\title{
Phylogenetic evidence for extensive horizontal gene transfer of type III secretion system genes among enterobacterial plant pathogens
}

\author{
Correspondence \\ Marianna Naum \\ marianna.naum@fda.hhs.gov
}

Received 9 April 2009

Revised 26 July 2009

Accepted 29 July 2009

\author{
Marianna Naum, ${ }^{1,2}$ Eric W. Brown ${ }^{2}$ and Roberta J. Mason-Gamer ${ }^{1}$ \\ ${ }^{1}$ Department of Biological Sciences, University of Illinois at Chicago, Chicago, IL 60611, USA \\ ${ }^{2}$ Division of Microbiology, Center for Food Safety and Applied Nutrition, Food and Drug \\ Administration (FDA), College Park, MD 20740, USA
}

\begin{abstract}
This study uses sequences from four genes, which are involved in the formation of the type III secretion apparatus, to determine the role of horizontal gene transfer in the evolution of virulence genes for the enterobacterial plant pathogens. Sequences of Erwinia, Brenneria, Pectobacterium, Dickeya and Pantoea were compared (a) with one another, (b) with sequences of enterobacterial animal pathogens, and (c) with sequences of plant pathogenic $\gamma$ and $\beta$ proteobacteria, to evaluate probable paths of lateral exchange leading to the current distribution of virulence determinants among these micro-organisms. Phylogenies were reconstructed based on $h r c C, h r c R, h r c J$ and hrcV gene sequences using parsimony and maximum-likelihood algorithms. Virulence gene phylogenies were also compared with several housekeeping gene loci in order to evaluate patterns of lateral versus vertical acquisition. The resulting phylogenies suggest that multiple horizontal gene transfer events have occurred both within and among the enterobacterial plant pathogens and plant pathogenic $\gamma$ and $\beta$ proteobacteria. hrc $J$ sequences are the most similar, exhibiting anywhere from 2 to $50 \%$ variation at the nucleotide level, with the highest degree of variation present between plant and animal pathogen sequences. $h r c V$ sequences are conserved among plant and animal pathogens at the $\mathrm{N}$ terminus. The $\mathrm{C}$-terminal domain is conserved only among the enterobacterial plant pathogens, as are the $h r c C$ and $h r c R$ sequences. Additionally, hrcJ and hrcV sequence phylogenies suggest that at least some type III secretion system virulence genes from enterobacterial plant pathogens are related more closely to those of the genus Pseudomonas, a conclusion neither supported nor refuted by $h r c C$ or $h r c R$.
\end{abstract}

\section{INTRODUCTION}

Type III secretion systems (T3SSs) are used by a wide array of plant and animal pathogenic Gram-negative bacteria to secrete and inject virulence factors directly into the cytosol of eukaryotic host cells (Galan \& Collmer, 1999; Madden et al., 2001). The T3SSs of Yersinia, Escherichia, Salmonella and Shigella have been extensively studied largely due to the potentially devastating health effects that these pathogens pose to human life (Galan \& Collmer, 1999; Groisman, 2001; Tampakaki et al., 2004). Interestingly, these same pathogenicity systems are also used by plant pathogens (e.g. Erwinia, Pseudomonas, Xanthomonas and

Abbreviations: GARLI, genetic algorithm for rapid likelihood inference; HGT, horizontal gene transfer; ILD, incongruence length difference; $M L$, maximum likelihood; MP, maximum parsimony; T3SS, type III secretion system.

The GenBank/EMBL/DDBJ accession numbers for the hrcC, hrcJ, hrcR and $h r c V$ sequences of the strains examined in this study are EU561240EU561268, EU561269-EU561288, EU561289-EU561316 and EU561317-EU561337, respectively.
Ralstonia) to infect numerous agriculturally important plants (Chen, 2006; Shumann, 1991; van der Zwet \& Beer, 1995), sometimes leading to millions of US dollars worth of losses each year among certain agricultural commodities.

T3SSs encode approximately 20-25 gene products, nine of which are conserved among plant and animal pathogens and are used in the formation of the needle complex, a unique T3SS structure (Fig. 1a) that is characteristic of this contact-dependent secretion mechanism (Bogdanove et al., 1996; Gophna et al., 2003). Of these nine conserved gene products, one is an outer membrane protein $(h r c C)$, one is periplasmic $(h r c J)$, likely serving as a connector of the inner and outer membrane components of the apparatus, four are integral inner membrane proteins $(h r c R, S, T$ and $U$ ) with periplasmic extensions, participating in the rod formation of the apparatus, and three are peripheral cytoplasmic proteins ( $h r c V, Q$ and $N$ ) involved in initiating secretion of effectors from the cytoplasm (Tampakaki et al., 2004). These nine structural components are so conserved among plant and animal pathogens that plant pathogenic 
(a)

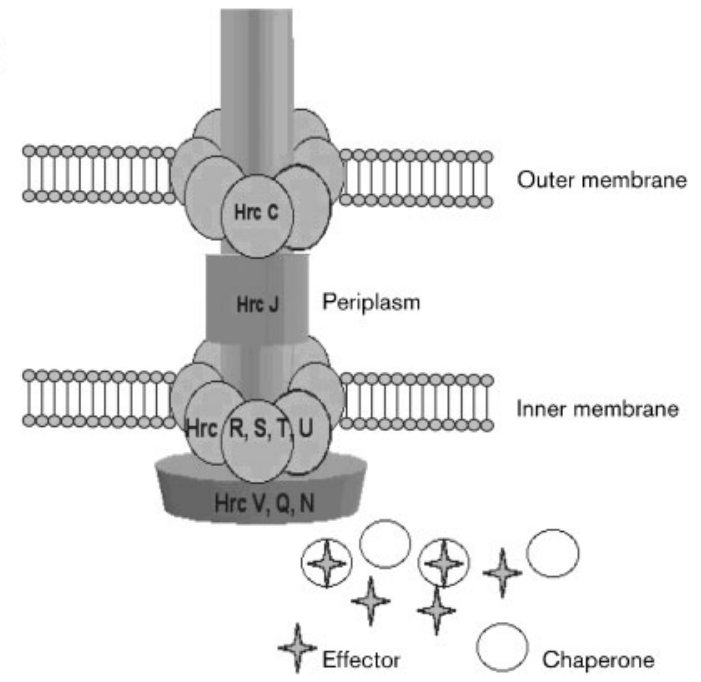

(b)

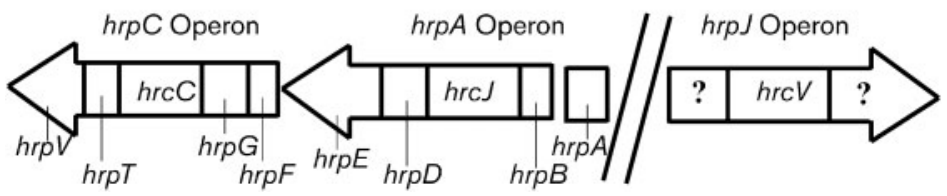

Fig. 1. (a) Schematic representation of the type III secretion needle complex used by Gram-negative plant and animal pathogenic micro-organisms. The four genes used to construct the phylogenies presented in this study are integral components of the needle complex, interacting with both the bacterial cell membranes and the chaperone-effector molecules secreted into the host cells. (b) Schematic representation of the characterized hrp/hrc operons as described for Erwinia amylovora (Oh et al., 2005). For visual clarity, oblique lines were added to the figure to denote the removal of T3SS chromosomal segments not related to the four hrc genes analysed here.
T3SS proteins of Xanthomonas have been shown to secrete virulence effectors (Yops) of Yersinia species in vitro (Rossier et al., 1999).

The T3SSs of both plant and animal pathogens are located in general on pathogenicity islands (PAIs), which appear to be horizontally transferred among disparate micro-organisms (Groisman \& Ochman, 1996). These PAIs are found mostly on plasmids among the enterobacterial animal pathogens. Conversely, they appear to have been integrated into the bacterial genomes of their enterobacterial plant pathogenic counterparts (Hacker et al., 1997; Oh et al., 2005; Toth et al., 2006). The pathogenicity island of Erwinia amylovora, which has been recently characterized (Oh et al., 2005), consists of a $62 \mathrm{~kb}$ region with 60 ORFs. A region of approximately $22 \mathrm{~kb}$ encodes the proteins of the T3SS in three operons: $h r p A, h r p C$ and $h r p J$ (Fig. 1b; Oh et al., 2005).

This study focuses on species of the genus Erwinia and the closely related genera Brenneria, Pectobacterium, Dickeya and Pantoea. This economically important group of enterobacterial plant pathogens is distributed worldwide, and they vary widely in their ecology, host range tropism, and pathogenicity (Krieg \& Holt, 1985). The strains used in this study have been well characterized taxonomically and their evolutionary history has been described based on numerous housekeeping genes (Brown et al., 2000; Hauben et al., 1998; Naum et al., 2008; Young \& Park, 2007). For example, Fig. 2(a) depicts a de novo evolutionary reconstruction of enterobacterial plant pathogenic species based on 16S rDNA gene sequences (Naum et al., 2008). To understand the role of horizontal gene transfer (HGT) of T3SS genes among the enterobacterial plant pathogens, we analysed sequences from four genes, $h r c C, h r c R, h r c J$ and $h r c V$. These four genes represent each of the three characterized operons (Oh et al., 2005), and encode proteins which serve different functional purposes (Fig. 1b).

\section{METHODS}

Sampling. A total of 29 strains were included in the phylogenetic analysis of the $h r c C$ sequences: 28 isolates were included in $h r c R$ and $h r c V$ analysis, and 52 samples were included in the $h r c J$ analysis (Table 1). Gene sequences for the enterobacterial plant pathogens were generated in our laboratory with the exception of the two Erwinia pyrifoliae sequences, which along with the remaining samples were downloaded from GenBank. The hrcJ dataset included the broadest sample of taxa because sequences for this gene were alignable across plant and animal pathogenic representatives. Strains of Erwinia, Brenneria, Pectobacterium, Dickeya and Pantoea were obtained from the American Type Culture Collection (ATCC), United States Department of Agriculture (USDA) (Appalachian Fruit Research Center, Kearneysville, WV), and from Dr Amy Charkowski (University of Wisconsin, Madison) as frozen cultures and LuriaBertani (LB) slants respectively. Sample authentication was confirmed based on 16S rDNA sequence analysis prior to their use (Naum et al., 2008).

Bacterial DNA extraction and PCR amplification. Total genomic DNA was extracted using a silica-based matrix (Instagene, Bio-Rad) as described in the manufacturer's directions, which allows for total chromosomal isolation from Gram-negative bacteria. Briefly, bacterial cells from $24 \mathrm{~h}$ cultures grown on LB agar at room temperature were resuspended in Instagene matrix and heated at $56{ }^{\circ} \mathrm{C}$ for $30 \mathrm{~min}$. 


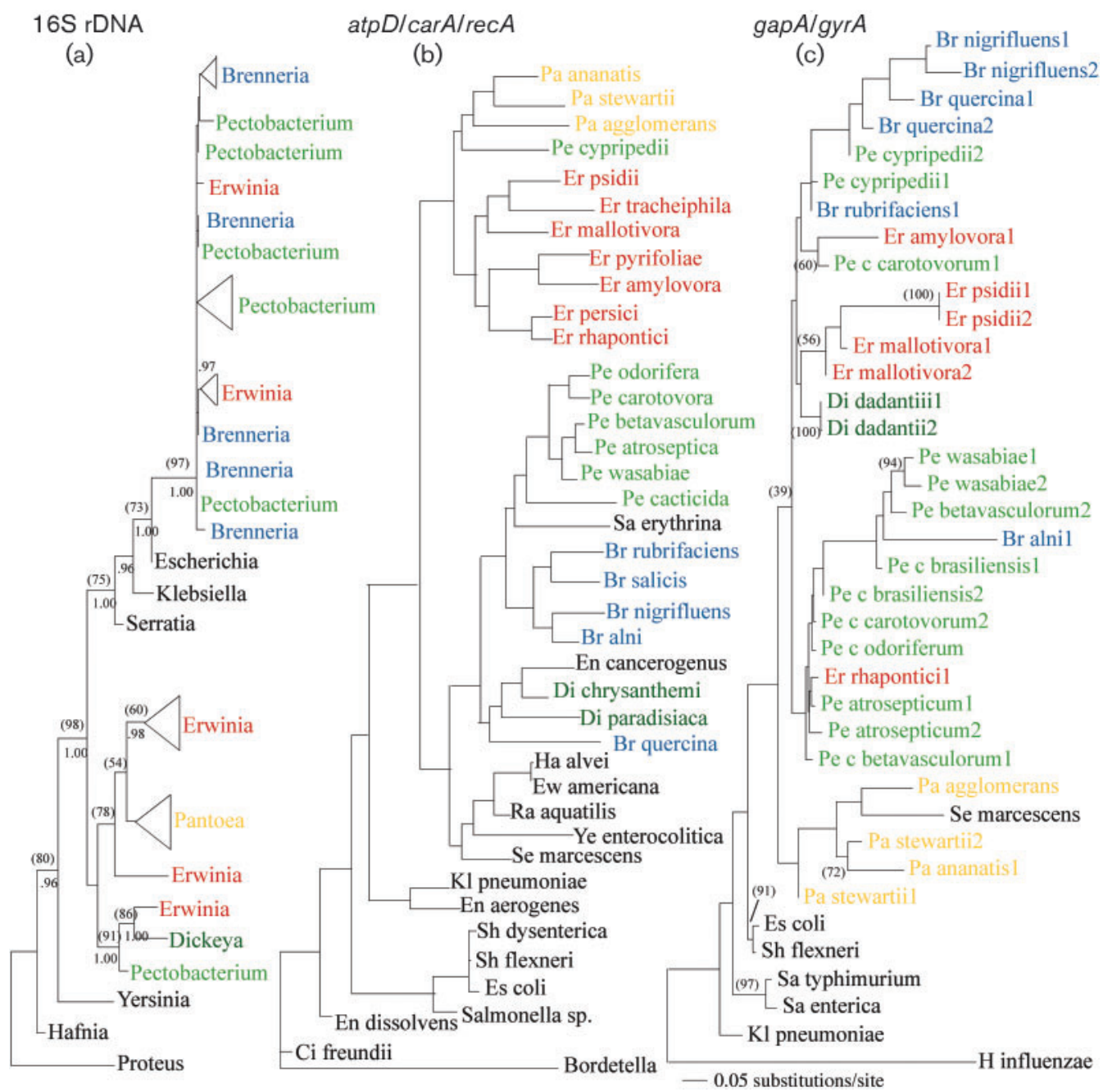

Fig. 2. (a) A de novo reconstructed model of a $16 \mathrm{~S}$ rDNA gene phylogeny encompassing the same enterobacterial plant pathogenic strains as those used in this study. To simplify this schematic representation we indicate only the genus and not the species names. (b) Redrawing of previously published relationships of the plant pathogenic enterobacteria based on a comparative analysis of concatenated alignments of atpD, carA and recA sequences using the likelihood algorithm (Young \& Park, 2007). (c) ML tree resulting from the phylogenetic analysis of the concatenated gapA/gyrA sequence dataset consisting of 899 nucleotide positions, produced in GARLI and viewed in PAUP*. ML bootstrap nodal support was generated in PAUP*, and subsequent values are reported for each node in parentheses. Abbreviations for the taxa used are as follows: Erwinia, Er; Brenneria, Br; Pectobacterium, Pe; Dickeya, Di; Pantoea, Pa; Citrobacter, Ci; Enterobacter, En; Escherichia, Es; Ewingella, Ew; Hafnia, Ha; Klebsiella, Kl; Photorhabdus, Ph; Proteus, Pr; Rhanella, Rh; Salmonella, Sa; Serratia, Se; Shigella, Sh; Xenorhabdus, Xe; Yersinia, Ye; Haemophilus, H. Multiple strains are denoted as 1 or 2 in the order that they have been listed in Table 3. For legibility purposes we have not italicized species names. Additionally, the plant pathogenic genera have been colour-coded for visual clarity, with Erwinia in red, Brenneria in blue, Pectobacterium in green, Pantoea in yellow and Dickeya in dark green.

The preparations were then vortexed vigorously and boiled for $10 \mathrm{~min}$. Suspensions were pelleted and the supernatant containing the DNA was decanted into a clean microtube. The optimum amount of DNA to be used in PCR amplification reactions was determined to be $2 \mu \mathrm{l}$, after an optimization PCR was carried out using 20,10, 5, 2 and $1 \mu \mathrm{l}$ of DNA. Oligonucleotide primers were designed for amplification of the $h r c J, h r c V, h r c C$ and $h r c R$ genes from conserved sequences flanking variable regions of the genes, using the few available Erwinia, Brenneria and Pectobacterium sequences from GenBank. The primers used were: $h r c C F\left(5^{\prime}-\mathrm{CTG}\right.$ GAG CAC CAT TTC CAG TGG T-3') and $h r c C R\left(5^{\prime}\right.$-CCT TCC AGC GCC TGG ATA TCG G-3'); $h r c R F\left(5^{\prime}-\right.$ CCA ATA TGG CGC TGT ACG GCA-3') and $h r c R R\left(5^{\prime}\right.$-GCC GTC GAG CAG SCG GGT CCA-3'); $h r c J F$ ( $5^{\prime}$-AAC GAT GCC AAC GAA G-3') and $h r c J R$ ( $5^{\prime}$-GGG ACA AAG ACA ATC GCC A-3'); and $h r c V F\left(5^{\prime}\right.$-CAT GAT CAT CAT TCC GCT GCC CA-3') and $h r c V R$ (5'-AGC TCT TGC GCC AGC TCT GGC TGT TCG CT-3'). PCR (Ehrlich et al., 1991) was carried out with $2 \mu$ DNA template, $2.5 \mu \mathrm{l}$ $10 \times$ PCR buffer (Sigma), $1.5 \mu \mathrm{l} 25 \mathrm{mM} \mathrm{MgCl}_{2}, 2 \mu \mathrm{l} 2.5 \mathrm{mM}$ dNTPs,

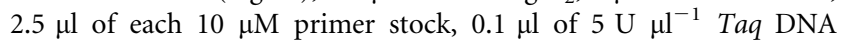
polymerase (Sigma), and purified distilled $\mathrm{H}_{2} \mathrm{O}$ for a total reaction 
Table 1. Bacterial strains and accession numbers for gene sequences used in the phylogenetic analyses

\begin{tabular}{|c|c|c|c|c|c|}
\hline \multirow[t]{2}{*}{ Taxon } & \multirow[t]{2}{*}{ Strain } & \multicolumn{4}{|c|}{ Accession number } \\
\hline & & hrcJ & $\operatorname{hrcV}$ & hrcC & hrcR \\
\hline \multicolumn{6}{|l|}{ Erwinia } \\
\hline Er, amylovora & 178 & EU561269 & EU561317 & EU561245 & EU561289 \\
\hline Er, amylovora & 552 & & L25828 & & \\
\hline Er. mallotivora & 8645 & EU561270 & EU561319 & EU561240 & EU561300 \\
\hline Er. mallotivora & 8646 & EU561271 & EU561320 & EU561246 & EU561308 \\
\hline Er. rhapontici & 1 & EU561272 & EU561321 & EU561243 & EU561292 \\
\hline Er. rhapontici & $29283^{*}$ & EU561273 & EU561322 & EU561244 & EU561293 \\
\hline Er. lupinicola & wl31 & EU561274 & EU561323 & EU561241 & EU561298 \\
\hline Er. lupinicola & 348 & EU561275 & & EU561242 & EU561299 \\
\hline Er. psidii & 8423 & EU561276 & EU561324 & EU561247 & EU561309 \\
\hline Er. psidii & 8429 & EU561277 & EU561325 & EU561248 & EU561310 \\
\hline Er. tracheiphila & 5845 & & & EU561253 & EU561306 \\
\hline Er. tracheiphila & $33245^{*}$ & & & & EU561307 \\
\hline Er. pyrifoliae & DSM12163 & DQ180962 & DQ180962 & & \\
\hline \multicolumn{6}{|l|}{ Brenneria } \\
\hline Br. nigrifluens & 1391 & EU561279 & EU561327 & EU561251 & EU561290 \\
\hline Br. nigrifluens & 4789 & EU561280 & EU561328 & EU561252 & EU561291 \\
\hline Br. alni & Pfv20* & EU561281 & & EU561255 & EU561303 \\
\hline Br. salicis & & & & EU561254 & \\
\hline Br. rubrifaciens & 9136 & & & EU561256 & EU561313 \\
\hline \multirow[t]{2}{*}{ Br. rubrifaciens } & 5950 & & & & EU561311 \\
\hline & $29291^{*}$ & & & EU561249 & EU561312 \\
\hline Br. quercina & 1846 & & & EU561250 & EU561301 \\
\hline Br. quercina & 1895 & & & & EU561302 \\
\hline \multicolumn{6}{|l|}{ Pectobacterium } \\
\hline \multicolumn{6}{|l|}{ Pe. carotovorum } \\
\hline subsp. carotovorum & 4 & EU561282 & EU561329 & EU561257 & EU561314 \\
\hline subsp. carotovorum & $15713^{*}$ & EU561283 & EU561330 & EU561258 & EU561297 \\
\hline subsp. brasiliensis & 1692 & EU561284 & EU561331 & EU561261 & EU561304 \\
\hline subsp. brasiliensis & 1695 & EU561285 & EU561332 & EU561262 & EU561305 \\
\hline subsp. odoriferum & SCRI 482 & EU561286 & EU561334 & EU561264 & EU561294 \\
\hline Pe. betavasculorum & Ecb1 & & & EU561259 & EU561295 \\
\hline Pe. betavasculorum & Ecb2 & & & EU561260 & EU56129 \\
\hline Pe. atrosepticum & SCRI 1043 & EU561287 & EU561333 & EU561267 & EU561316 \\
\hline \multicolumn{6}{|l|}{ Dickeya } \\
\hline Di. dadantii & $11663^{*}$ & & EU561335 & EU561265 & EU561315 \\
\hline Di. dadantii & EC16 & & & EU561266 & \\
\hline \multicolumn{6}{|l|}{ Pantoea } \\
\hline Pa. agglomerans & $33243^{*}$ & & EU561336 & & \\
\hline Pa. stewartii & 8199 & EU561288 & EU561337 & EU561268 & \\
\hline \multicolumn{6}{|l|}{ Pseudomonas } \\
\hline Ps. syringae & B728a & NC_007005 & NC_007005 & & \\
\hline Ps. syringae 1 & $1448 \mathrm{~A}$ & NC_005773 & & & \\
\hline Ps. syringae 2 & DC3000 & AE016853 & & & \\
\hline Ps. syringae 3 & LMG5090 & DQ246442 & & & \\
\hline Ps. syringae 4 & ATCC115288 & AB049570 & & & \\
\hline Ps. mendocina & ymp & СР000680 & & & \\
\hline Ps. cichorii & $83-1$ & DQ168848 & & & \\
\hline Ps. viridiflava & RMX3.1b & AY597283 & & & \\
\hline Ps. viridiflava & RMX23.1a & AY597282 & & & \\
\hline Ps. viridiflava & ME3.1b & AY597281 & & & \\
\hline \multicolumn{6}{|l|}{ Xanthomonas } \\
\hline Xa. campestris & pv. vesicatora & AM039952 & AM039952 & & \\
\hline Xa. campestris 1 & $85-10$ & NC_007508 & & & \\
\hline Xa. campestris 2 & 80004 & NC_007086 & & & \\
\hline
\end{tabular}


Table 1. cont.

\begin{tabular}{|c|c|c|c|c|c|}
\hline \multirow[t]{2}{*}{ Taxon } & \multirow[t]{2}{*}{ Strain } & \multicolumn{4}{|c|}{ Accession number } \\
\hline & & hrcJ & $h r c V$ & hrcC & hrcR \\
\hline Xa. axonopodis & 306 & NC_003919 & & & \\
\hline Xa. oryzae 1 & BLS256 & AY875714 & & & \\
\hline Xa. oryzae 2 & MAFF311018 & AP008229 & & & \\
\hline Ra. solanacearum & GMI1000 & AL646053 & AL646053 & & \\
\hline Ra. solanacearum 1 & IPO1609 & AJ245811 & & & \\
\hline Ra. solanacearum 2 & & NC_003296 & & & \\
\hline \multicolumn{6}{|l|}{ Escherichia } \\
\hline Es. coli & 042 & AF200363 & AF200363 & & \\
\hline \multicolumn{6}{|l|}{ Salmonella } \\
\hline Sa. enterica & SC-B67 & NC_006905 & & & \\
\hline Sa. enterica 1 & CT18 & AL627271 & & & \\
\hline Sa. typhimurium & LT2 & NC_003197 & NC_003197 & & \\
\hline \multicolumn{6}{|l|}{ Yersinia } \\
\hline Ye. pestis & CO92 & AL117189 & & & \\
\hline Ye. enterocolitica & 8081 & AM286415 & & & \\
\hline
\end{tabular}

${ }^{\star}$ Indicates the type culture for each of the species represented as originally obtained from ATCC.

volume of $25 \mu$ l. PCR was performed in a PTC-200 thermal cycler (MJ Research) under the following conditions: initial denaturation at $94{ }^{\circ} \mathrm{C}$ for $2 \mathrm{~min}, 94{ }^{\circ} \mathrm{C}$ for $50 \mathrm{~s}, 58{ }^{\circ} \mathrm{C}$ for $50 \mathrm{~s}$ and $72{ }^{\circ} \mathrm{C}$ for $45 \mathrm{~s}(35$ cycles), ending with incubation at $72{ }^{\circ} \mathrm{C}$ for $8 \mathrm{~min}$. The amplified segments were $\sim 850 \mathrm{bp}$ long for $h r c C, \sim 500 \mathrm{bp}$ long for $h r c R$, $\sim 500 \mathrm{bp}$ long for $h r c J$, and $\sim 1500 \mathrm{bp}$ long for $h r c V$.

Cloning and DNA sequencing. PCR products were purified using QIAquick gel extraction kits (Qiagen) according to the manufacturer's protocol, except that DNA was eluted in $20 \mu \mathrm{l}$ buffer. Cloning reactions were completed using pGEM T Easy cloning kits (Promega) according to the manufacturer's protocol, except that the ligation and transformation reaction volumes were halved. Cloned inserts were amplified directly from colonies using the original PCR primers and amplification conditions. PCR products were cleaned using exonuclease I and shrimp alkaline phosphatase as in Mason-Gamer (2004). Purified PCR products were sequenced in both directions using an automated Sanger dideoxy-chain-termination method (Amplicon Express). DNA sequences were edited and combined in Sequencher v.4.1 (Gene Codes Corporation).

Sequence alignment and phylogenetic analyses. DNA sequences and resultant computer-translated amino acid sequences ( $h r c C$ and $h r c R$ ) were aligned using CLUSTAL W v.1.5 (Thompson et al., 1994), followed by manual adjustments in MacClade v.4.0 (Maddison \& Maddison, 2000). Amino acid codon coloration was used in MacClade v.4.0 (Maddison \& Maddison, 2000) on the nucleotide datasets to ensure the resulting alignments did not alter the protein sequence. Regions judged to have an ambiguous alignment were excluded ( $\sim 400 \mathrm{bp}$ for $h r c C, 45 \%$; $120 \mathrm{bp}$ for $h r c R, 21 \%$; and $\sim 590 \mathrm{bp}$ for $h r c V, 40 \%$ ) prior to the phylogenetic analyses. All datasets were first analysed in PAUP ${ }^{*}$ (Swofford, 2001) using the principle of maximum parsimony (MP) under heuristic search methods using tree bisection-reconnection (TBR) branch-swapping, and 100 random taxon addition replicates. For the nucleotide datasets, the shortest trees were then used as the starting topology for the evaluation of 16 nested models of sequence evolution (Frati et al., 1997; Sullivan et al., 1997; Swofford et al., 1996). The best-fit model of substitution for each dataset was determined by the likelihood score of each under a chi-squared approximation of the null distribution (Yang et al., 1995). Parameter space was searched for the best tree with simultaneous estimation for model parameters using a maximum likelihood (ML) search conducted using the Genetic Algorithm for Rapid Likelihood Inference (GARLI) software (Zwickl, 2006). As recommended by Zwickl (2006), multiple runs were performed $(\sim 20)$ to ensure that results were consistent, as the algorithm is inherently stochastic. The log-likelihood values of each run were retained in order to compare the individual runs, and the tree and model parameters corresponding to the best score were used.

Branch support was determined in GARLI by 100 ML bootstrap iterations (Felsenstein, 1985), and with Bayesian posterior probability (MrBayes v.3.1.2) approximation of 1000000 generations, discarding $25 \%$ (2500) of the tree samples as recommended in the MrBayes v.3.1.2 manual (Huelsenbeck \& Ronquist, 2001). For Bayesian character support methods, parameters of sequence evolution estimated from the final ML tree were used (Table 2). Amino acid character support was determined in PAUP* by 1000 bootstrap iterations and 1000000 generations of Bayesian (MrBayes v.3.1.2; (Huelsenbeck \& Ronquist, 2001) posterior probability approximation under the principle of MP, again discarding $25 \%$ (2500) of the tree samples. 
Table 2. ML model parameters used in the phylogenetic analyses of the nucleotide data

\begin{tabular}{|c|c|c|c|c|}
\hline Gene & Model $^{\star}$ & Base frequencies & Rate matrix & $\mathbf{I}+\Gamma$ \\
\hline$h r c J$ & $\mathrm{GTR}+\Gamma$ & $\begin{array}{l}\text { A,C,G,T }(0.226665,0.271862 \\
0.305593,0.195877)\end{array}$ & $\begin{array}{c}\text { AC,AG,AT,CG,CT,GT }(1.05305,2.34379 \\
0.63654,0.97192,3.64812,1.00000)\end{array}$ & NA, 0.692249 \\
\hline$h r c V$ & $\mathrm{GTR}+\mathrm{I}+\Gamma$ & $\begin{array}{l}\mathrm{A}, \mathrm{C}, \mathrm{G}, \mathrm{T}(0.185737,0.305335 \\
0.285268,0.223658)\end{array}$ & $\begin{array}{c}\text { AC,AG,AT,CG,CT,GT }(1.75130,4.17764 \\
1.35511,1.40323,5.02710,1.00000)\end{array}$ & $0.017862,0.43826$ \\
\hline$h r c C$ & $\mathrm{GTR}+\mathrm{I}+\Gamma$ & $\begin{array}{l}\mathrm{A}, \mathrm{C}, \mathrm{G}, \mathrm{T}(0.213890,0.295173 \\
0.287391,0.203006)\end{array}$ & $\begin{array}{c}\text { AC,AG,AT,CG,CT,GT }(1.48399,3.94858 \\
0.71867,1.03759,7.14812,1.00000)\end{array}$ & $0.190209,0.672602$ \\
\hline$h r c R$ & $\mathrm{GTR}+\mathrm{I}+\Gamma$ & $\begin{array}{l}\mathrm{A}, \mathrm{C}, \mathrm{G}, \mathrm{T}(0.212377,0.305330 \\
0.243388,0.238905)\end{array}$ & $\begin{array}{c}\text { AC,AG,AT,CG,CT,GT }(1.48844,4.45970 \\
0.79636,2.22929,5.79619,1.00000)\end{array}$ & $0.346505,1.601213$ \\
\hline
\end{tabular}

${ }^{\star}$ GTR, general time reversible; I, proportion of invariable sites; $\Gamma$, number of gamma distribution rate categories; NA, not available.

Nucleotide gene trees were rooted by midpoint rooting, rather than outgroup rooting (Schuettpelz \& Hoot, 2006), because the homologous gene sequences from the closest available outgroups were too divergent to align reliably. The $h r c C$ and $h r c R$ amino acid sequence trees were rooted with Escherichia coli (GenBank accession numbers AJ303141 and AF022236) and Yersinia pestis (GenBank accession number NC_004839), respectively. The absence of suitable outgroup taxa impeded the detection of the direction of certain HGT events between and among plant and animal pathogenic taxa. However, in these specific instances, direction was addressed inferentially.

Housekeeping gene analysis for determination of strain evolution. To determine phylogenetic incongruence in relation to HGT we included three housekeeping gene trees in this study in order to provide a vertically inherited evolutionary framework to which $h r c$ gene phylogenies were compared. Fig. 2 shows a de novo reconstructed model of a $16 \mathrm{~S}$ rDNA gene phylogeny encompassing the same enterobacterial plant pathogenic strains used in this study, with only the genus names listed to simplify this schematic representation (Naum et al., 2008). Fig. 2(b) shows a redrawing of the concatenated housekeeping gene phylogeny presented by Young \& Park (2007) based on atpD, carA and recA gene sequences, with representatives of the same Erwinia, Brenneria, Pectobacterium, Dickeya and Pantoea species used in this study. Additionally, Fig. 2 (c) is the phylogeny resulting from a concatenated analysis of gapA and gyrA gene sequences including 31 plant pathogen taxa, six animal pathogen representatives and one outgroup taxon (Table 3; Hauben $e t$ al., 1998; Lelliott \& Dickey, 1985; Starr \& Chatterjee, 1972). Primers used for PCR amplification were gapAF (5'-TGA AAT ATG ACT CCA CTC ACG G-3'), gapAR ( $5^{\prime}$-TAG AGG ACG GGA TGA TGT TCT G-3'), gyrAF ( $5^{\prime}$-GCT GCG CGY CCW GAG GCT GGA C-3') and gyrAR (5'-CCA CGR CTG GCT TCC GGC A-3'), and amplification reactions were carried out following the protocol described above. The amplified segments were $\sim 410 \mathrm{bp}$ for gapA and $\sim 489$ bp for gyrA. DNA sequencing and phylogenetic analyses were carried out as described above, with the concatenated dataset encompassing 899 nucleotide positions.

Incongruence length difference (ILD) test. Statistical congruence between genes was assessed using the ILD test (Farris et al., 1994) available in PAUP* (Swofford, 2001). Gene partitions were assembled as nexus files in interleaved format using the PAUP ${ }^{*}$ text editor, such that each gene represented a single interleaved portion of the combined data matrix. In this fashion, data partitions were easily defined as the beginning and end of interleaved blocks of sequences. ILD comparisons were performed using the partition homogeneity test on the plant pathogenic strains shared among all eight gene datasets ( $h r c J, h r c V, h r c C, h r c R$, 16S rDNA, gapA, gyrA and gapA/
gyrA), with 1000 data partitions using heuristic searches (Allard et al., 1999)

\section{RESULTS}

The hrcC DNA alignment consisted of 29 taxa and 855 characters, of which 458 were informative, whereas the amino acid alignment consisted of 31 taxa, including the outgroups, and 291 characters of which 150 were informative. The final ML search of the nucleotide data resulted in one tree (Fig. 3a), and the (most parsimonious) MP analysis of the amino acid data yielded 16 equally most parsimonious trees $[272$ steps; consistency index $(\mathrm{CI})=0.87$; rescaled consistency $(\mathrm{RC})=0.81]$, from which the strict consensus was derived (Fig. 3b). The hrcR DNA alignment consisted of 28 taxa and 508 characters, of which 384 were informative, while the amino acid alignment consisted of 29 taxa and 170 characters, of which 133 were informative. The final ML search of the nucleotide data resulted in one tree (Fig. 4a), and the MP analysis of the amino acid data yielded two equally most parsimonious trees (175 steps; $\mathrm{CI}=0.93 ; \mathrm{RC}=0.97)$, from which a strict consensus was derived (Fig. 4b). The hrcJ alignment consisted of 52 taxa and 454 characters after truncating the beginning and end of the dataset in order to eliminate gaps resulting from the absence of data due to varying sequence lengths. The GARLI tree with the best loglikelihood score for $h r c J$ is presented in Fig. 5(a). The $h r c V$ alignment consisted of 28 taxa and 1536 characters, of which only the first 910 were used in the phylogenetic analysis because the remaining 630 base pairs could only be meaningfully aligned for the enterobacterial plant pathogens. After the 800th base pair approximately, the sequences of Pseudomonas, Xanthomonas, Ralstonia, Escherichia, Salmonella and Yersinia began diverging from the sequences of Erwinia, Brenneria, Pectobacterium, Dickeya and Pantoea, and by the 910th nucleotide position, they could no longer be aligned. The GARLI tree with the best score for $h r c V$ is presented in Fig. 5(b).

The concatenated gapA/gyrA alignment consisted of 38 taxa and 899 characters after truncating the beginning and end of the dataset in order to eliminate gaps resulting from the 
Table 3. Bacterial strains and accession numbers for gene sequences used in the housekeeping gene phylogenetic analyses

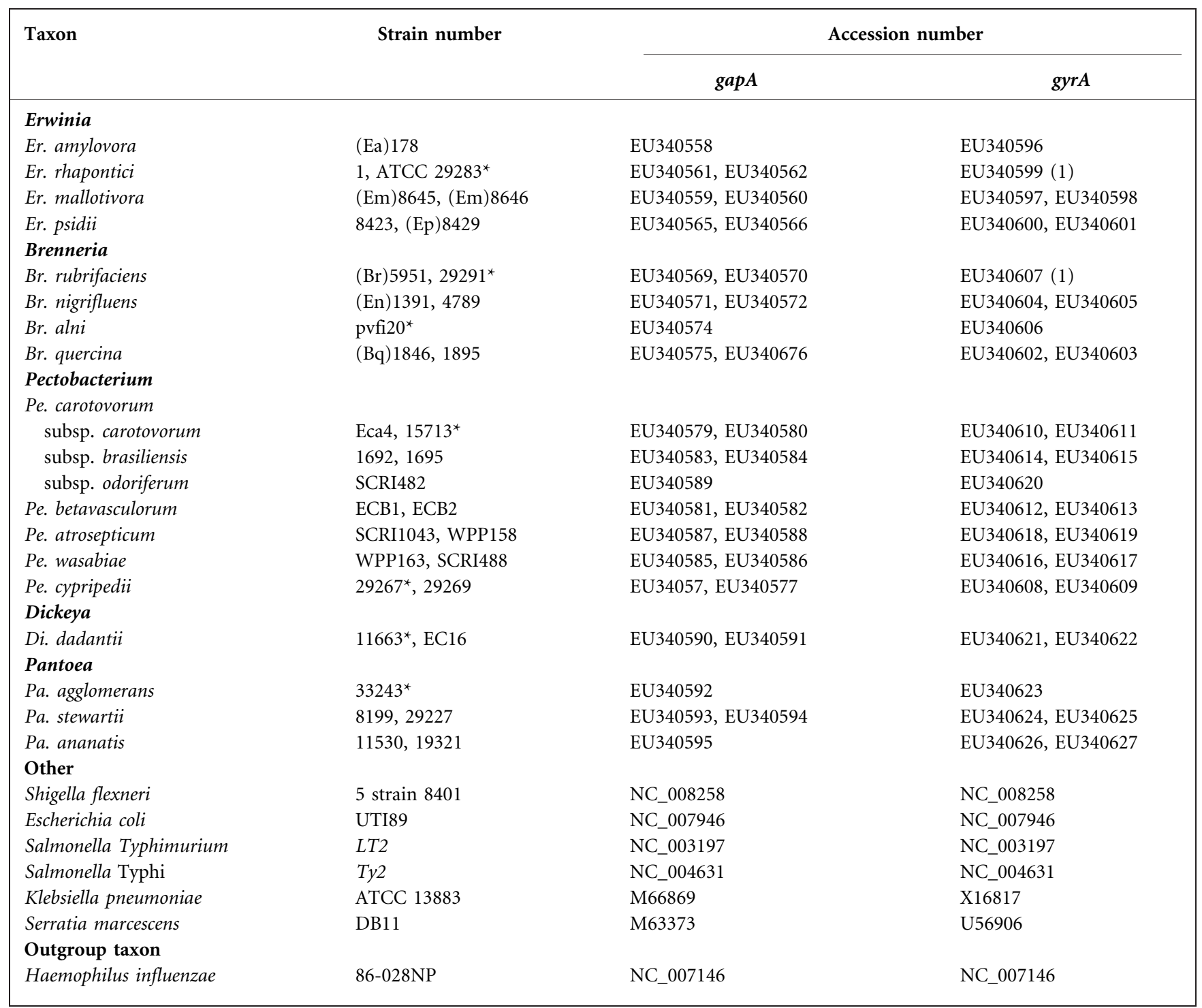

${ }^{\star}$ Indicates the type culture for each of the species represented as originally obtained from ATCC.

absence of data due to varying sequence lengths. The GARLI tree with the best log-likelihood score for gapA/ gyrA is presented in Fig. 2(c).

Erwinia, Brenneria and Pectobacterium are non-monophyletic on the $h r c C$ and $h r c R$ phylogenies, a conclusion strongly supported by clades C and D (Figs $3 a$ and $4 a$ ). Both clades contain multiple Erwinia, Brenneria and Pectobacterium taxa with bootstrap support values of 90 and $98 \%$ for clades $\mathrm{C}$ and $\mathrm{D}$, respectively. In addition, strains of Erwinia mallotivora 1 and 2, Pectobacterium carotovorum subsp. carotovorum 1 and 2, and Pectobacterium betavasculorum 1 and 2 are broken up, each being more closely related to other Erwinia and Pectobacterium species than to each other. We resequenced $h r c C$ and $h r c R$ for Erwinia tracheiphila 1 and Brenneria salicis 1 to confirm that the original sequences were not artefacts, since in the $h r c C$ phylogeny, the sequences are indistinguishable and on a long branch (Fig. 3a). Even though the $h r c C$ and $h r c R$ reconstructions include only enterobacterial plant pathogen representatives, we suggest a monophyletic origin of the virulence determinants comprising their T3SS because of the extensive divergence between the sequences of Erwinia, Brenneria, Pectobacterium, Dickeya and Pantoea from sequences of the enterobacterial animal pathogens and plant pathogenic $\gamma$ and $\beta$ proteobacteria, at both the nucleotide and the amino acid levels.

The $h r c J$ and $h r c V$ trees (Fig. 5) are generally congruent (Table 4), indicating a substantial degree of evolutionary and genetic linkage between these loci. The relationships are largely unaffected by the number of representative sequences, which differs between the two phylogenies 


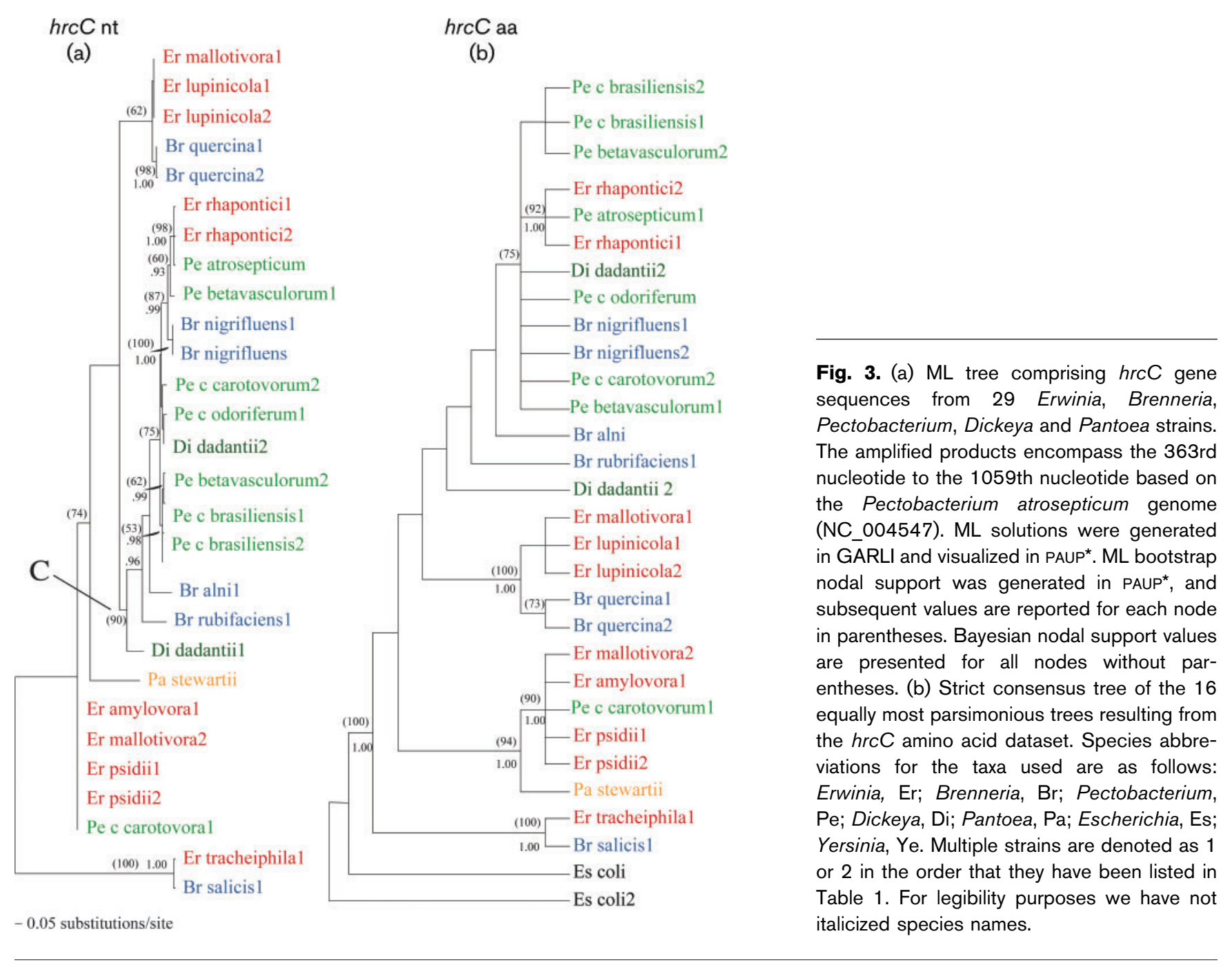

because $h r c J$ sequences were more conserved, allowing us to include a greater number of animal pathogen and $\gamma$ and $\beta$ proteobacterial plant pathogen representatives. Both trees place Xanthomonas and Ralstonia as sisters to one another and to the other plant pathogens, and they also group Escherichia and Salmonella together, and as a sister group to Yersinia. Moreover, the trees differentiate the plant and animal pathogens into two well-defined clades. Furthermore, both phylogenies group Erwinia, Brenneria, Pectobacterium, Dickeya and Pantoea as closest relatives, with Pseudomonas syringae as their sister group. Additionally, the enterobacterial plant pathogens form two well-supported clades, designated A and B (Fig. 5a). Clade A consists of Pectobacterium and Brenneria species, along with two strains of Erwinia rhapontici, while clade B is dominated by Erwinia and Pantoea. However, in the $h r c V$ tree (Fig. 5b), Erwinia lupinicola 1 is placed outside both clade A and clade B (although this position is not well supported), while P. carotovorum subsp. carotovorum 1 is found in clade B. Taken together, these results suggest that the $h r c J$ and $h r c V$ virulence genes of the enterobacterial plant pathogens and Pseudomonas share a recent common ancestor.

Furthermore, the $h r c J$ and $h r c V$ trees are congruent to one another with respect to the resulting relationships between the enterobacterial plant pathogens (Table 4). The trees are also consistent in the placement of Erwinia, Brenneria, Pectobacterium, Dickeya and Pantoea with the other plant pathogens and not with the enteric animal pathogens, a result in agreement with and expanding on a previous study of hrcJ sequences (Brown et al., 1998). It is notable that the $h r c C$ and $h r c R$ trees are incongruent to one another and to both the $h r c J$ and $h r c V$ phylogenies (Table 4), which could be the result of multiple interspecific HGT events among the enterobacterial plant pathogens after the acquisition of the T3SS into this lineage.

On the other hand, all of the housekeeping gene trees (Fig. 2) support multiple independent origins of the plant pathogenic genera within the Enterobacteriaceae, and all loci are consistent in their taxonomic classification of the four genera. Additionally, in all three housekeeping gene 


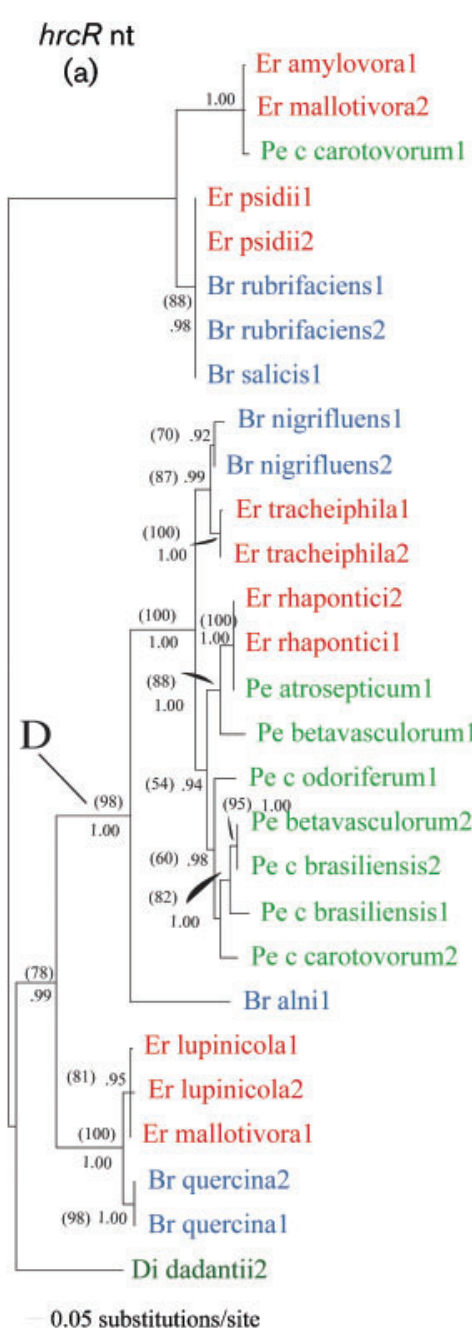

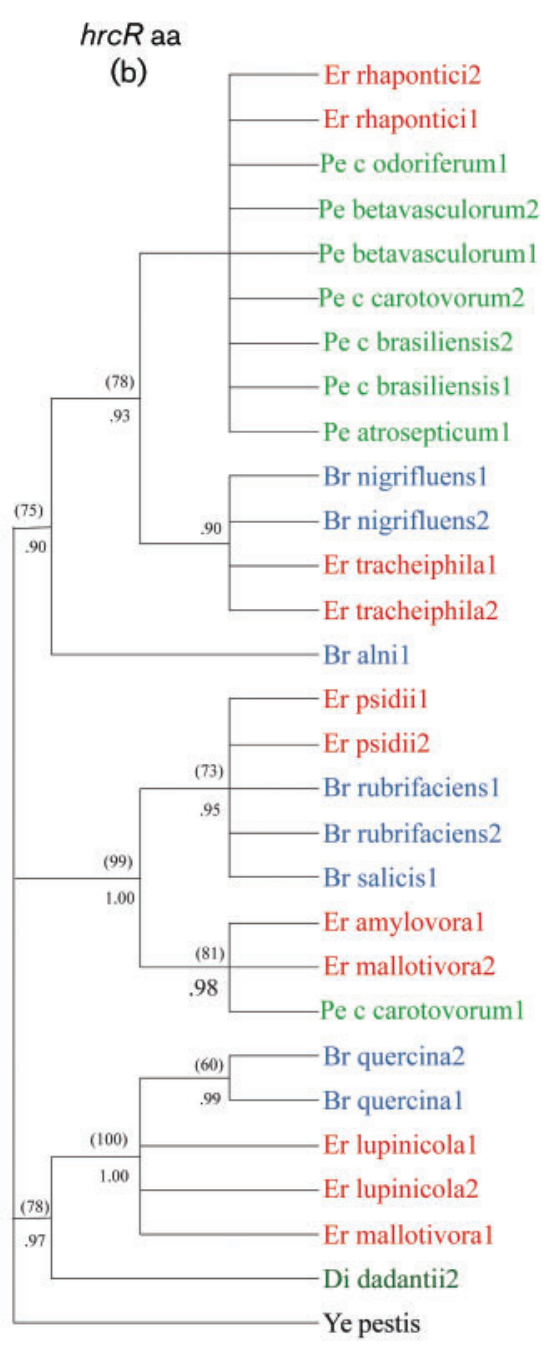

Fig. 4. (a) $M L$ tree comprising $h r c R$ gene sequences from 28 enterobacterial plant pathogen species representatives. The amplified products encompass the 210th nucleotide to the 630th nucleotide based on the Pectobacterium atrosepticum genome (NC_004547). ML solutions were generated in GARLI and visualized in PAUP*. ML bootstrap nodal support was generated in PAUP*, and subsequent values are reported for each node in parentheses. Bayesian nodal support values are presented for all nodes without parentheses. (b) Strict consensus tree of the two equally most parsimonious trees resulting from the $h r c R$ amino acid dataset. Species abbreviations for the taxa used are as follows: Erwinia, Er; Brenneria, Br; Pectobacterium, Pe; Dickeya, Di; Pantoea, Pa; Escherichia, Es; Yersinia, Ye. Multiple strains are denoted as 1 or 2 in the order that they have been listed in Table 1. For legibility purposes we have not italicized species names. phylogenies, it is evident that housekeeping gene evolution - taken here to depict the vertical evolution of the strains analysed - contrasts with virulence gene evolution and does not support a single origin of a plant pathogenic ancestor followed by speciation into the numerous plant pathogenic genera and species.

Finally, ILD testing comparing the virulence genes with one another and with several housekeeping loci confirmed the hypothesis of incongruence ( $P=0.001$, Table 4$)$. Strikingly, each pairwise comparison of the virulence genes with the housekeeping loci resulted in a statistically significant $P$ value $(P<0.05$, Table 4$)$, leading us to reject the null hypothesis. This result indicates that the virulence determinants examined in this study have all been subject to some level of lateral transfer between the enterobacterial plant pathogens. The only gene dataset comparison for which we could not reject the null hypothesis (i.e. for which the dataset partitions were found to be congruent based on the ILD test) was between the $h r c J$ and $h r c V$ partitions. These results support our hypothesis that both ancestral and recent HGT events have been driving the evolution of virulence in the enterobacterial plant pathogens.

\section{DISCUSSION}

The nine proteins involved in forming the needle complex of the T3SS are conserved across multiple animal and plant pathogens and are believed to share a common ancestor with the flagellar export mechanism used by many microorganisms (Gophna et al., 2003). However, it is evident from the results of this study that vertical acquisition (Fig. 2) has been interjected by numerous HGT events, which undeniably have been a driving force in shaping the evolutionary history of the enterobacterial plant pathogen virulence genes (Figs 3, 4 and 5).

Despite the sampling differences between the $h r c J$ and $h r c V$ datasets, analyses of the conserved gene segments resulted in congruent phylogenies for the two genes (Fig. 5, Table 4 ), which are in conflict with the phylogenies generated from $h r c C$ and $h r c R$ gene sequences (Figs 3 and 4, Table 4). Unlike the $h r c J$ and $h r c V$ datasets, the partial $h r c C$ and $h r c R$ 


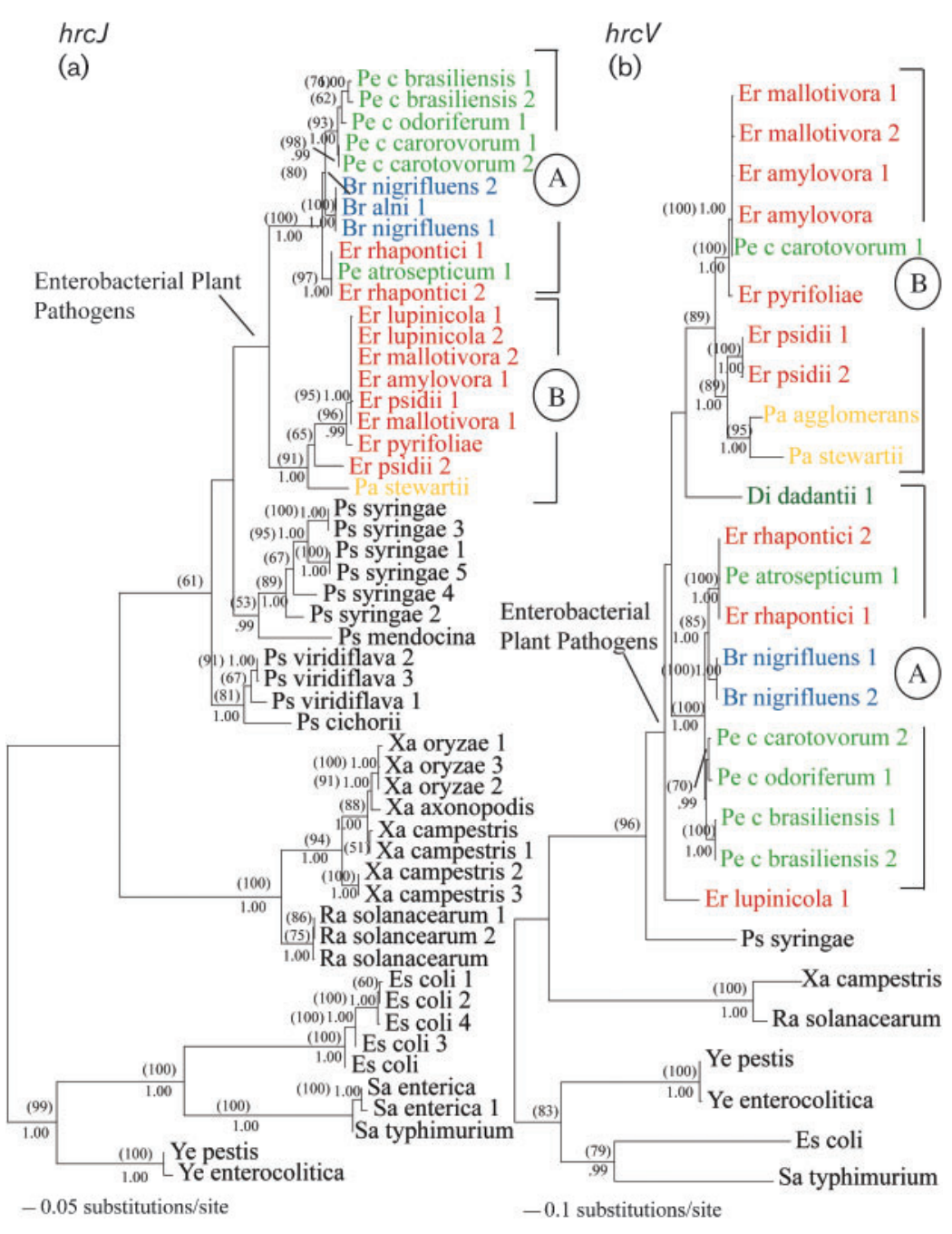

Fig. 5. (a) ML tree resulting from the phylogenetic analysis of the $h r c J$ sequence dataset including enterobacterial plant and animal pathogenic representatives, and non-enterobacterial plant pathogenic $\gamma$ and $\beta$ proteobacterial taxa. The amplified products encompass the 93rd nucleotide to the 414th nucleotide based on the Pectobacterium atrosepticum genome (NC_004547). (b) ML tree resulting from the phylogenetic analysis of the $h r c V$ sequence dataset. The amplified products encompass the 124th nucleotide to the 1359th nucleotide of the Pectobacterium atrosepticum genome (NC_004547), with only the first 910 nucleotides (124-1034) included in the analyses. The resultant maximum trees for these datasets were produced in GARLI and viewed in PAUP*. ML bootstrap nodal support was generated in PAUP*, and subsequent values are reported for each node in parentheses. Bayesian nodal support values are presented for all nodes without parentheses. Species abbreviations for the taxa used are as follows: Erwinia, Er; Brenneria, Br; Pectobacterium, Pe; Dickeya, Di; Pantoea, Pa; Pseudomonas, Ps; Xanthomonas, Xa; Ralstonia, Ra; Escherichia, Es; Salmonella, Sa; Yersinia, Ye. Multiple strains are denoted as 1 or 2 in the order that they have been listed in Table 1. For legibility purposes we have not italicized species names. gene sequences we studied were highly variable, much like the C-terminal region of $h r c V$, and only sequences from Erwinia, Brenneria, Pectobacterium, Dickeya and Pantoea could be meaningfully aligned. In addition, because the taxonomic placement of Erwinia, Brenneria, Pectobacterium, Dickeya and Pantoea in the family Enterobacteriaceae has been confirmed by analysis of multiple housekeeping genes (Fig. 2; Brown et al., 2000;

Table 4. Pair-wise ILD values among eight virulence loci in 12 representative enterobacterial plant pathogens

\begin{tabular}{|llllllll|}
\hline \multirow{2}{*}{ Locus } & \multicolumn{7}{c|}{ P value (1000 partitions) } \\
\cline { 2 - 8 } & hrcV & hrcC & hrcR & $\begin{array}{c}\text { 16S } \\
\text { rDNA }\end{array}$ & gapA & gyrA & $\begin{array}{c}\text { gapA/ } \\
\text { gryA }\end{array}$ \\
\hline hrcJ & 0.174 & 0.001 & 0.001 & 0.001 & 0.001 & 0.001 & 0.001 \\
$h r c V$ & & 0.001 & 0.001 & 0.001 & 0.001 & 0.001 & 0.001 \\
$h r c C$ & & & 0.001 & 0.001 & 0.001 & 0.001 & 0.001 \\
$h r c R$ & & & & 0.001 & 0.001 & 0.001 & 0.001 \\
\hline
\end{tabular}

Hauben et al., 1998; Young \& Park, 2007), it is striking that the $h r c J$ and $h r c V$ trees (Fig. 5) reveal that the enterobacterial plant pathogens likely acquired their $h r c J$ and $h r c V$ genes during a single HGT event from the same ancestor as Pseudomonas syringae prior to their speciation. Assessing the evolutionary history of $h r c C$ and $h r c R$ gene sequences has, however, been a more difficult task. First, there are relatively few $h r c C$ and $h r c R$ homologues currently in the nucleotide databases (GenBank and EMBL), and obtaining an appropriately broad sample from throughout the family would have been outside the scope of this study. Second, based on available sequences, it is evident that homologous gene sequences from the enterobacterial animal pathogens as well as other plant pathogenic $\gamma$ and $\beta$ proteobacteria are so divergent from those of Erwinia, Brenneria, Pectobacterium and Dickeya that they cannot be meaningfully compared in a phylogenetic context. This extensive divergence is interpreted here as important, albeit indirect, evidence that the T3SS determinants of the enterobacterial plant pathogens together form a monophyletic group. Virulence sequences from within the enterobacterial phytopathogens are far 
more similar to one another than they are to any available sequences from animal and other plant pathogenic taxa.

A conclusion of monophyly, however, assumes a singular evolutionary origin for the enterobacterial plant pathogens that is not supported by the currently accepted taxonomy of the group (Fig. 2). The $16 \mathrm{~S} \mathrm{rDNA}$, atpD/carA/recA and gapA/gyrA phylogenies suggest that the enterobacterial plant pathogenic genera of Erwinia, Brenneria, Pectobacterium, Dickeya and Pantoea arose independently multiple times within the family. Therefore, if we recognize that the hypothesis of polyphyly accurately reflects the evolutionary history of Erwinia, Brenneria, Pectobacterium, Dickeya and Pantoea then there must be an alternative explanation for the relationships resulting from the virulence gene phylogenies. For example, if plant pathogenicity evolved independently multiple times and was associated with a reduced rate of virulence gene evolution in the resulting lineages, then we would expect the plant pathogens to exhibit relatively high sequence similarity in spite of their polyphyletic origins. Conversely, if an animal pathogenic lineage has arisen from within a lineage of plant pathogens, and the host switch was associated with an increased rate of sequence evolution, then eventually the plant pathogens will display greater sequence similarity to one another than to the animal pathogen in spite of their paraphyly relative to the derived animal pathogen. However, both of these scenarios would require rate changes that affect $h r c C$ and $h r c R$ simultaneously and in the same way, while at the same time $h r c J$ and $h r c V$ remain unaffected. These hypotheses would be more plausible if $h r c C$ and $h r c R$ encoded extracellular proteins, as it has been suggested that extracellular protein components undergo bursts of rapid polypeptide evolution to facilitate the mechanical adaptations necessary for the T3SS to interface with different eukaryotic cell types (Dale \& Moran, 2006). However, since neither gene product is known to be expressed extracellularly, these explanations seem unlikely.

Alternatively, repeated exchanges of common T3SS alleles among the enteric plant pathogens could provide a similar phylogenetic outcome - that is one of monophyly - among the T3SS genes used in this study. Clearly, the repeated horizontal transfer of a few preferred alleles would result in the homogenization of population structure and subsequently result in the evolutionary divergence of the phytopathogens from closely related animal pathogenic neighbours that lie just beyond the barrier of these extensive allelic exchanges. The effects of continued interspecific HGT are clearly seen in the differing phylogenetic placement of the bacterial strains from within species among the phylogenies (Figs 3, 4 and 5). There are cases where strains of a species group together on one tree, but are separated on the other tree, suggesting that one of the two strains has acquired a phylogenetically distinct gene copy through HGT. For example, the two E. mallotivora sequences are nearly identical, and group together, on the $h r c J$ and $h r c V$ trees (Fig. 5), but they are widely separated from one another on the $h r c C$ and $h r c R$ trees (Figs 3 and 4). The two P. carotovorum subsp. carotovorum strains, on the other hand, group closely together on the $h r c J$ tree, but are widely separated on the $h r c C$ (Fig. 3a), hrcR (Fig. 4a) and $h r c V$ (Fig. 5b) trees. Conflicts involving interspecific relationships are evident as well. For example, the grouping of E. tracheiphila 1 with $B$. salicis 1 at the base of the hrcC tree (Fig. 3a) is in conflict with both of their positions on the $h r c R$ tree (Fig. 4a) (neither is included on the $h r c J$ or $h r c V$ tree). A likely explanation for the conflicting placements of these bacterial strains and species is the acquisition of different gene copies. Since the strains were isolated from different individuals of the same host species, and transfer events are believed to be influenced more by physical proximity of strains than by phylogenetic relatedness (Matte-Tailliez et al., 2002; Toth et al., 2006), it is possible that the bacterial strains acquired their copies of the genes from neighbouring bacteria on the same plant.

Finally, even though the lack of an appropriate outgroup has encumbered our ability to detect the direction of the noted HGT events, we can infer that the enterobacterial plant pathogens probably acquired their T3SS prior to their radiative speciation, from the same ancestor as Pseudomonas syringae, a conclusion that is strengthened by the observed \% GC content, which is uniform between these four virulence genes and housekeeping genes such as $16 \mathrm{~S}$ rDNA, gapA and gyrA. Thus, it is ironic and somewhat unexpected that the virulence genes may have provided an additional and important clue to buttress the hypothesis of a monophyletic plant pathogenic lineage in the family Enterobacteriaceae, as originally proposed by Brown et al. (2000). Moreover, the differences in the phylogenetic positions of the enterobacterial plant pathogenic species among the $h r c J, h r c V, h r c C$ and $h r c R$ trees are possibly the result of continued HGT events followed by genetic recombination during and possibly after the speciation of Erwinia, Brenneria, Pectobacterium, Dickeya and Pantoea, as clearly seen in the phylogenetic placement of the enterobacterial plant pathogenic strains. Additionally, the ineffectiveness of the flagellar gene homologues as outgroups corroborates the hypothesis proposed by Gophna et al. (2003) stating that the T3SS and the flagellar export mechanism share a common ancestor but have evolved independently of one another since. Taken together, these results have provided important clues about the natural history of acquisition of virulence among a highly significant group of bacterial plant pathogens, and although the precept that HGT has forged virulence evolution is certainly not a novel one (Brown et al., 1998; Cohan, 1996; Comas et al., 2006; Dale \& Moran, 2006; Gophna et al., 2003), we know of no other analysis encompassing the enterobacterial plant pathogens which has demonstrated that the diversification of the virulence operons is likely to be ancestral to the radiation of the plant pathogens themselves. The monophyly of the T3SS genes of the enterobacterial phytopathogens is evidenced by the extraordinary observed diversification at the amino acid 
level (in $h r c C$, $h r c R$ and $h r c V$ ), which was so advanced that the inclusion of relevant enteric homologues was largely an impossible task. This diversification among the plant and animal pathogens within the Enterobactericaceae has likely persisted for epochs among these unique lineages, and continues to be maintained by ongoing HGT events.

\section{ACKNOWLEDGEMENTS}

Thanks to A. Charkowski of the University of Wisconsin, Madison, for very generously providing the Pectobacterium strains used in this study, and to Diane McCarthy, Michael Jorgensen and Keith Lampel for valuable comments on an earlier version of the manuscript. Also, a special thanks to Peter Naum and Sergio Mojica for helping us create the illustration in Fig. 1. The research was supported by National Science Foundation (NSF) grant number DEB 0426194 to R. J. M.-G., and a University of Illinois at Chicago (UIC) Provosts Award to M. N.

\section{REFERENCES}

Allard, M. W., Farris, J. S. \& Carpenter, J. (1999). Congruence among mammalian mitochondrial genes. Cladistics 15, 75-84.

Bogdanove, A. J., Beer, S. V., Bonas, U., Boucher, C. A., Collmer, A., Coplin, D. L., Cornelis, G. R., Huang, H. C., Hutcheson, S. W. \& other authors (1996). Unified nomenclature for broadly conserved hrp genes of phytopathogenic bacteria. Mol Microbiol 20, 681-683.

Brown, E. W., Allard, M. W. \& van der Zwet, T. (1998). Phylogenetic characterization of the eubacterial $\operatorname{lcrD}$ gene family: molecular evolutionary aspects of pathogen induced hypersensitivity in plants. Cladistics 14, 45-62.

Brown, E. W., Davis, R. M., Gouk, C. \& van der Zwet, T. (2000). Phylogenetic relationships of necrogenic Erwinia and Brenneria species as revealed by glyceraldehyde-3-phosphate dehydrogenase gene sequences. Int J Syst Evol Microbiol 50, 2057-2068.

Chen, L. L. (2006). Identification of genomic islands in six plant pathogens. Gene 374, 134-141.

Cohan, F. M. (1996). The role of genetic exchange in bacterial evolution. ASM News 62, 631-636.

Comas, I., Moya, A., Azad, R. K., Lawrence, J. G. \& GonzalezCandelas, F. (2006). The evolutionary origin of Xanthomonadales genomes and the nature of the horizontal gene transfer process. $\mathrm{Mol}$ Biol Evol 23, 2049-2057.

Dale, C. \& Moran, N. A. (2006). Molecular interactions between bacterial symbionts and their hosts. Cell 126, 453-465.

Ehrlich, H. A., Gelford, D. \& Snisky, J. J. (1991). Recent advances in the polymerase chain reaction. Science 252, 1643.

Farris, J. S., Kallersjo, M., Kluge, A. G. \& Bult, C. (1994). Testing significance of incongruence. Cladistics 10, 315-319.

Felsenstein, J. (1985). Confidence limits on phylogenies: an approach using the bootstrap. Evolution 39, 783-791.

Frati, F., Simon, C., Sullivan, J. \& Swofford, D. L. (1997). Evolution of the mitochondrial cytochrome oxidase II gene in Collembola. $\mathrm{J} \mathrm{Mol}$ Evol 44, 145-158.

Galan, J. E. \& Collmer, A. (1999). Type III secretion machines: bacterial devices for protein delivery into host cells. Science 284, 1322-1328.

Gophna, U., Ron, E. Z. \& Graur, D. (2003). Bacterial type III secretion systems are ancient and evolved by multiple horizontal-transfer events. Gene 312, 151-163.
Groisman, E. A. (2001). Principles of Bacterial Pathogenesis. New York: Academic Press.

Groisman, E. A. \& Ochman, H. (1996). Pathogenicity islands: bacterial evolution in quantum leaps. Cell 87, 791-794.

Hacker, J., Blum-Oehler, G., Muhldorfer, I. \& Tschape, H. (1997). Pathogenicity islands of virulent bacteria: structure, function, and impact on microbial evolution. Mol Microbiol 23, 1089-1097.

Hauben, L., Moore, E. R. B., Vauterin, L., Steenackers, L., Mergaert, J., Verdonck, L. \& Swings, J. (1998). Phylogenetic position of phytopathogens within the Enterobacteriaceae. Syst Appl Microbiol 21, 384-397.

Huelsenbeck, J. P. \& Ronquist, F. (2001). MRBAYES: Bayesian inference of phylogenetic trees. Bioinformatics 17, 754-755.

Krieg, N. R. \& Holt, J. G. (1985). Bergey's Manual of Systematic Bacteriology. New York: Williams and Wilkins.

Lelliott, R. A. \& Dickey, R. S. (1985). Genus VII. Erwinia. In Bergey's Manual of Systematic Bacteriology, pp. 469-476. Edited by N. R. Krieg \& J. G. Holt. New York: Williams and Wilkins.

Madden, J. C., Ruiz, N. \& Caparon, M. (2001). Cytolysin-mediated translocation (CMT): a functional equivalent of type III secretion in Gram-positive bacteria. Cell 104, 143-152.

Maddison, W. P. \& Maddison, D. R. (2000). MacClade. Sunderland, MA: Sinauer Associates.

Mason-Gamer, R. J. (2004). Reticulate evolution, introgression, and intertribal gene capture in an allohexaploid grass. Syst Biol 53, 25-37.

Matte-Tailliez, O., Brochier, C., Forterre, P. \& Philippe, H. (2002). Archaeal phylogeny based on ribosomal proteins. Mol Biol Evol 19, 631-639.

Naum, M., Mason-Gamer, R. J. \& Brown, E. W. (2008). Is $16 \mathrm{~S}$ rDNA a reliable phylogenetic marker to characterize relationships below the family level in the Enterobacteriaceae? J Mol Evol 66, 630-642.

Oh, C. S., Kim, J. F. \& Beer, S. V. (2005). The Hrp pathogenicity island of Erwinia amylovora and identification of three novel genes required for systemic infection. Mol Plant Pathol 6, 125-138.

Rossier, O., Wengelnik, K., Hahn, K. \& Bonas, U. (1999). The Xanthomonas Hrp type III system secretes proteins from plant and mammalian bacterial pathogens. Proc Natl Acad Sci U S A 96, 9368-9373.

Schuettpelz, E. \& Hoot, S. B. (2006). Inferring the root of Isoetes: exploring alternatives in the absence of an acceptable outgroup. Syst Bot 31, 258-270.

Shumann, G. L. (1991). Plant Diseases: their Biology and Social Impact. St. Paul, MN: American Phytopathology Society.

Starr, M. P. \& Chatterjee, A. K. (1972). The genus Erwinia: enterobacteria pathogenic to plants and animals. Annu Rev Microbiol 26, 389-426.

Sullivan, J., Markert, J. A. \& Kilpatrick, C. W. (1997). Phylogeography and molecular systematics of the Peromyscus aztecus species group (Rodentia: Muridae) inferred using parsimony and likelihood. Syst Biol 46, 426-440.

Swofford, D. L. (2001). PAUP* Phylogenetic analysis using parsimony (*and other methods), version 4b10. Sunderland, MA: Sinauer Associates.

Swofford, D. L., Olsen, G. J., Waddell, P. J. \& Hillis, D. M. (1996). Phylogenetic inference. In Molecular Systematics, pp. 407-514. Edited by D. M. Hillis \& C. Moritz. Sunderland, MA: Sinauer Associates.

Tampakaki, A. P., Fadouloglou, V. E., Gazi, A. D., Panopoulos, N. J. \& Kokkinidis, M. (2004). Conserved features of type III secretion. Cell Microbiol 6, 805-816.

Thompson, J. D., Higgins, D. \& Gibson, T. J. (1994). CLUSTAL version W: a novel multiple sequence alignment program. Nucleic Acids Res 22, 4673-4680. 
Toth, I. K., Pritchard, L. \& Birch, P. R. J. (2006). Comparative genomics reveals what makes an enterobacterial plant pathogen. Annu Rev Phytopathol 44, 305-336.

van der Zwet, T. \& Beer, S. V. (1995). Fire Blight-its Nature, Prevention, and Control: a Practical Guide to Integrated Disease Management. USDA Agricultural Information Bulletin number 631, 83 pp.

Yang, Z., Goldman, N. \& Friday, A. (1995). Maximum-likelihood trees from DNA sequences: a peculiar statistical estimation problem. Syst Biol 44, 384-399.
Young, J. M. \& Park, D. C. (2007). Relationships of plant pathogenic enterobacteria based on partial atpD, carA, and $\operatorname{rec} A$ as individual and concatenated nucleotide and peptide sequences. Syst Appl Microbiol 30, 343-354.

Zwickl, D. (2006). Genetic algorithm approaches for the phylogenetic analysis of large biological sequence datasets under the maximum likelihood criterion. $\mathrm{PhD}$ thesis. University of Texas, Austin.

Edited by: I. K. Toth 